\title{
PRÓTESE OVERLAY NO PACIENTE COM PERDA DE DIMENSÃO VERTICAL CAUSADA PELO BRUXISMO: Experiência de estágio clínico
}

DOI: $10.22289 / 2446-922 X . V 3 N 1 A 2$

\author{
Samira de Leles \\ Mariana de Melo Peres \\ Ana Luiza Serralha de Veloso \\ Roberto Wagner Lopes Góes \\ Fernando Nascimento \\ Lia Dietrich ${ }^{1}$
}

\section{RESUMO}

Hábitos parafuncionais como o bruxismo podem causar diminuição da dimensão vertical de oclusão em consequência dos desgastes severos nos dentes, sendo necessário o restabelecimento da DVO antes de qualquer procedimento permanente. As próteses parciais do tipo overlay são indicadas nesses casos como alternativa de tratamento. Este trabalho mostra um caso clínico que tem como objetivo relatar uma reabilitação com próteses parciais removíveis provisórias do tipo overlay para restabelecimento da DVO e logo após foi realizado um tratamento reabilitador final. O resultado desse trabalho demonstrou a eficácia do uso de prótese parcial removível provisória do tipo overlay nos casos em que há perda da DVO relacionada aos hábitos parafuncionais.

Palavras-chave: Overlay. Reabilitação oral. Dimensão vertical.

\footnotetext{
${ }^{1}$ Endereço eletrônico de contato: lia_dietrich@yahoo.com.br
}

Recebido em 04/05/2017. Aprovado pelo Conselho Editorial e aceito para publicação em 07/06/2017.

Rev. Psicol Saúde e Debate. Jul., 2017:3(1):12-21. 


\section{ABSTRACT}

Parafunctional habits such as bruxism cause decreased vertical dimension of occlusion causing severe wear on the teeth, requiring the restoration of DVO before any permanent procedure. The partial dentures of the overlay type are indicated in such cases as an alternative treatment. This study aims to report a rehabilitation with temporary removable partial dentures overlay type reestablishing the DVO and after being held a definitive treatment. The end result of this study demonstrated the efficacy of provisional RPD overlay type where there is loss of DVO.

Keywords: Overlay. Oral rehabilitation. Vertical dimension.

\section{INTRODUÇÃO}

O desgaste dentário é visto como uma situação patológica quando excessivo para a idade do paciente, exigindo tratamento por questões funcionais ou cosméticas. A diminuição exagerada da dimensão vertical de oclusão (DVO) normalmente é causado em decorrência de bruxismo intenso, sendo associada ao estresse emocional, distúrbios do sono e alterações do sistema nervoso central. ${ }^{(1)}$

Vários fatores etiológicos podem ocasionar desgastes dentais severos: ausência dos dentes posteriores que irá ocasionar o deslizamento anterior da mandíbula, promovendo perda elevada de estrutura dentária dos dentes anteriores; hábitos parafuncionais (interposição de objetos rígidos entre os dentes ou bruxismo). ${ }^{(2)}$

A palavra bruxismo origina-se do grego "bruchein" que quer dizer apertamento, fricção ou atrito dos dentes sem efeito funcional. Também é caracterizado como contato estático ou dinâmico da oclusão, através de movimentos laterais e protusivos, normalmente durante o sono de forma inconsciente apresentando-se em forma de "apertamento" ou "ranger" de dentes. Sua etiologia ainda não é bem compreendida tratando-se de uma desordem complexa e multifatorial. ${ }^{(3,4)}$

Em situações que ocorreram perdas dentárias ou excessivo desgastes dos dentes, é necessário haver um restabelecimento da DVO antes de ser realizado qualquer procedimento restaurador definitivo. A DVO é determinada de acordo com a posição vertical da mandíbula em relação a maxila quando os elementos dentários superiores e inferiores estão em máxima intercuspidação. Quando há comprometimento da DVO deverá ser realizado modificações oclusais ao longo da reabilitação oral, devendo ser avaliado a 
estética, função, fonética e conforto do paciente. Sendo indicação para o tratamento dessa situação a instalação prévia de uma overlay. ${ }^{(5,6,7)}$

A prótese parcial removível tipo overlay é utilizada em reabilitações orais onde há desgaste dentário excessivo e necessidade de restabelecimento da dimensão vertical de oclusão (DVO). Essa diminuição da DVO pode ser gerada por diversas causas dentre elas estão os hábitos parafuncionais. ${ }^{(8)}$

Essas próteses também podem ser utilizadas no tratamento que antecede a cirurgia ortognática, melhoria do plano oclusal, tratamento de pacientes com defeitos congênitos, condicionamento do músculo e no restabelecimento da DVO, servindo como orientação para uma posterior reabilitação. ${ }^{(9) .}$

Ela deve ser confeccionada a partir de um enceramento e sua finalização ocorre através de um processo de prensagem de resina acrílica. Após a instalação a prótese contribui para restabelecer a DVO obtendo estabilidade oclusal, sendo adaptada tanto em relação cêntrica quanto em lateralidade direita e esquerda, assim restabelecendo integralmente a função, contribuindo para o conforto do paciente e melhoria da sua capacidade mastigatória, devolvendo a saúde e integridade dos arcos dentários. ${ }^{(2,6)}$

As próteses parciais tipo overlay são classificadas de acordo com o material usado para envolver a oclusal, como também de acordo com a função. Quanto à sua estrutura podem ser classificadas como metálicas, de resina, de porcelana, ou mista que é a combinação de metálico e estético. As próteses de estrutura metálica possuem a desvantagem de não suportar os impactos prejudicando assim o periodonto. Quando confeccionada de resina é maior a chance de descolamento, fratura, descoloração e desgaste do material que recobre a incisal ou oclusal. Quanto à função podem ser divididas em provisória e definitiva. ${ }^{(8,9)}$

Dentre as vantagens relacionadas à alternativa de tratamento temos: a facilidade de reparo, facilidade de higienização, baixo custo, dentes remanescentes não necessitam de desgastes, e reversibilidade. Dentre as desvantagens estão: dificuldade de fala e possível desconforto devido à dificuldade de adaptação da prótese, técnica laboratorial complexa, e complexidade do processo laboratorial. ${ }^{(1)}$

A falta ou o desgaste exagerado dos dentes anteriores influenciam diretamente a estética e a harmonia do sorriso, que são fundamentais para o bem-estar do paciente. $(2,10)$

Rev. Psicol Saúde e Debate. Jul., 2017:3(1):12-21. 
Este trabalho tem como objetivo relatar uma experiência de estágio clínico realizado na Clínica de Estágio da Faculdade Patos de Minas de um tratamento executado em um paciente com perda de dimensão vertical de oclusão causado por hábito parafuncional, e teve como finalidade o restabelecimento da DVO com prótese parcial removível do tipo overlay, devolvendo estética parcial, fonação e função ao paciente reabilitado.

\section{METODOLOGIA E RELATO DE EXPERIÊNCIA DE ESTÁGIO}

\section{CLÍNICO}

Paciente E. R. J., gênero feminino, 55 anos, fumante há 35 anos compareceu à Clínica Integrada de Odontologia da Faculdade Patos de Minas queixando-se de desgaste excessivo nos dentes, diastemas e várias ausências dentárias. Na ocasião a paciente não fazia uso de prótese parcial removível. Durante a anamnese a paciente relatou que era portadora de bruxismo noturno. No exame clínico foi constatado que a paciente possui edentulismo parcial em ambas arcadas, podendo ser classificada como classe I de Kennedy superior e inferior, os dentes remanescentes apresentavam desgaste nas faces oclusais e incisais das superfícies dentárias dos dentes 11, 12, 13, 21, 22, 23, 34, 33, 32, 31, 41, 42 e 43, e diminuição da DVO. (Figuras 1 e 2) ) Realizou a mensuração da dimensão vertical de oclusão com associação dos métodos métrico, fonético e da deglutição, com o paciente em relação cêntrica. Foi verificado então perda excessiva da DVO.

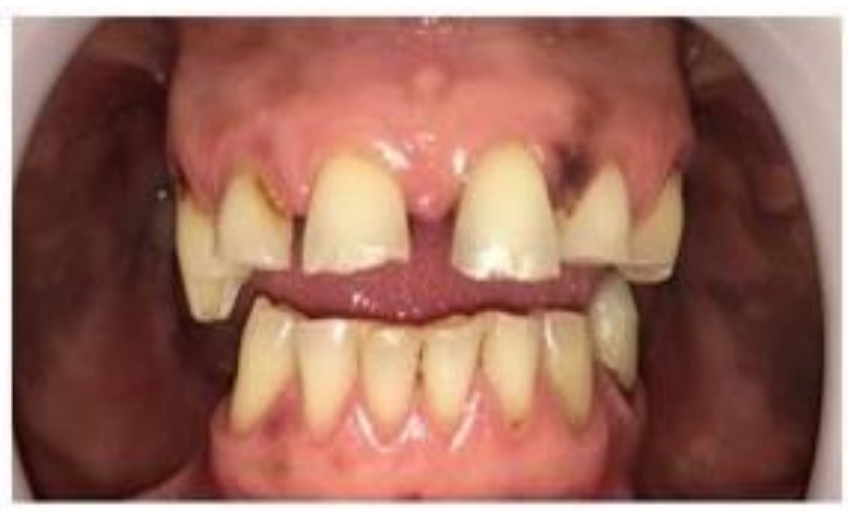

Figura 1 Foto intraoral mostrando situação inicial do paciente

Rev. Psicol Saúde e Debate. Jul., 2017:3(1):12-21. 


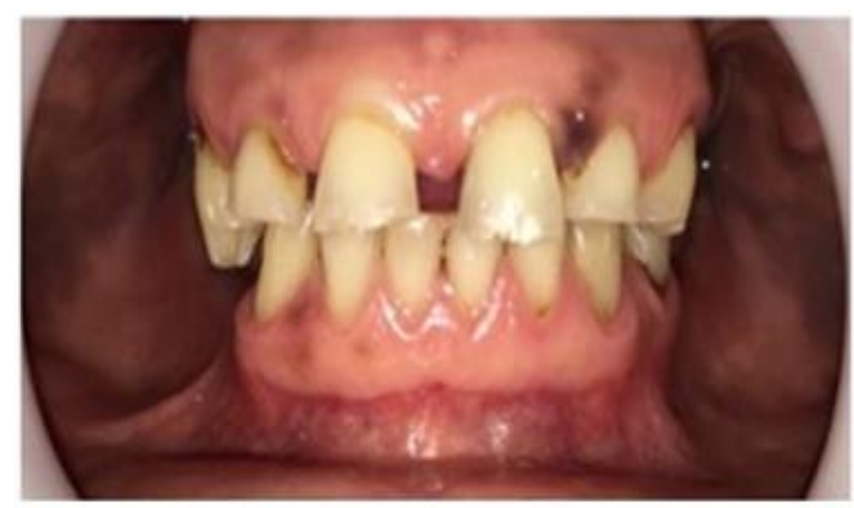

Figura 2 Foto intraoral mostrando situação inicial do paciente

Após a realização da anamnese e exame clínico, radiografias e fotografias, realizouse a moldagem das arcadas superior e inferior para obtenção dos modelos de estudo e posteriormente montagem em articulador semi ajustável (ASA) com registro em arco facial para que fosse possível identificar as discrepâncias oclusais e a perda da DVO. Após a montagem em ASA, análise e avaliação do caso, foi traçado um plano de tratamento, onde iniciou-se pela confecção de um dispositivo desprogramador neuromuscular "JIG" com o paciente manipulado em relação cêntrica. Posteriormente realizou-se a prova dos dentes em boca com a base já ajustada, observando estética, fonética, oclusão e bem estar para a paciente. Ao harmonizar a base de prova, foi conduzido para as etapas laboratoriais para posterior instalação. Em seguida confeccionou uma placa de resina acrílica provisória sobre a superfície oclusal dos dentes remanescentes, aumentando assim a DVO. (Figuras 3, 4 e 5)

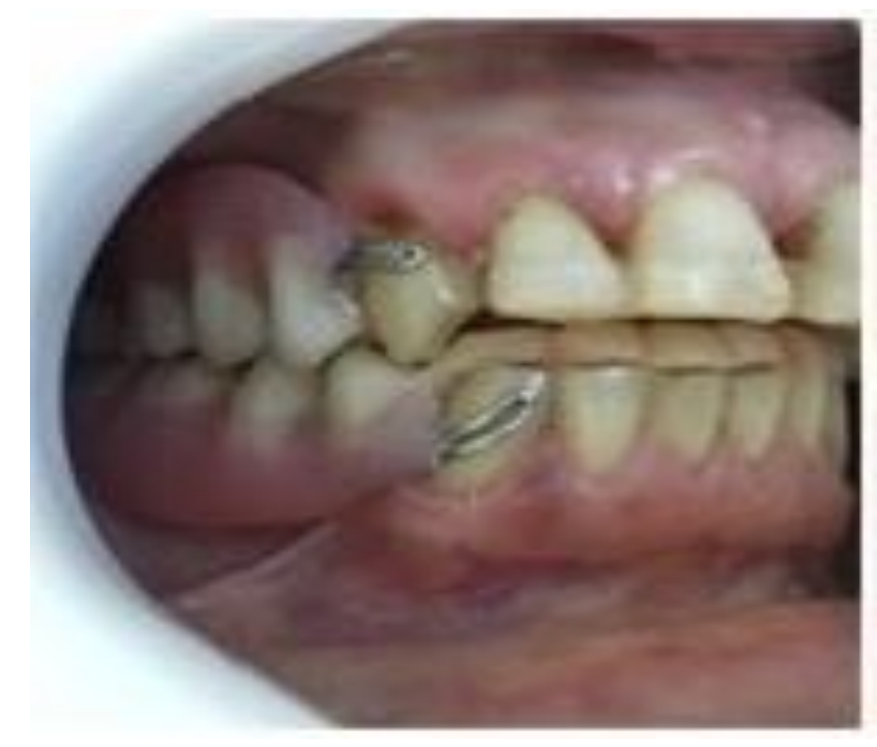

Figura 3 Foto intraoral em vista lateral mostrando a prova da overlay antes do reembasamento final.

Rev. Psicol Saúde e Debate. Jul., 2017:3(1):12-21. 


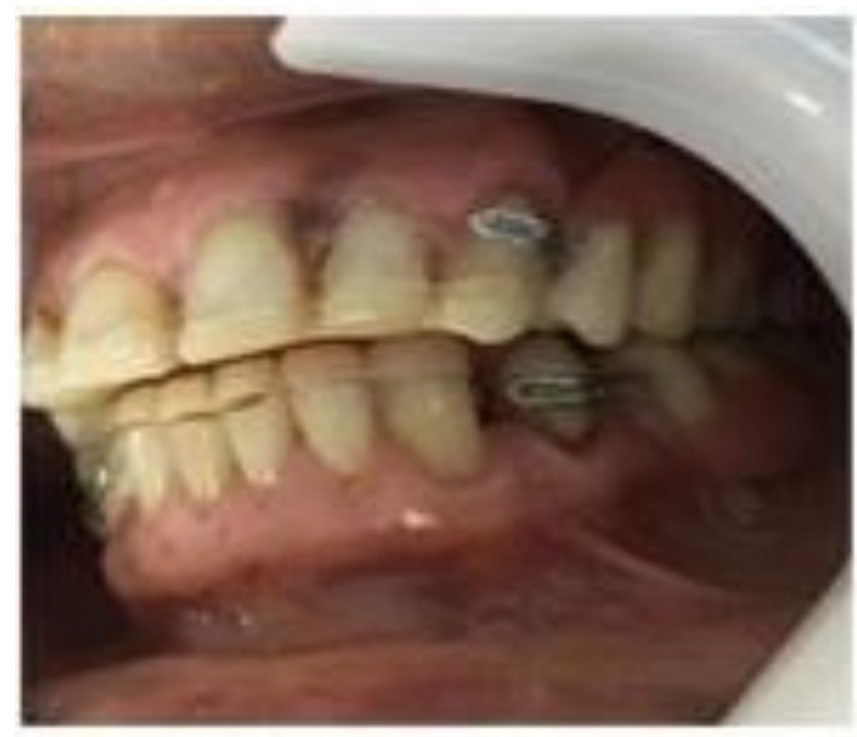

Figura 4 Foto intraoral em vista lateral mostrando a prova da overlay antes do reembasamento final.

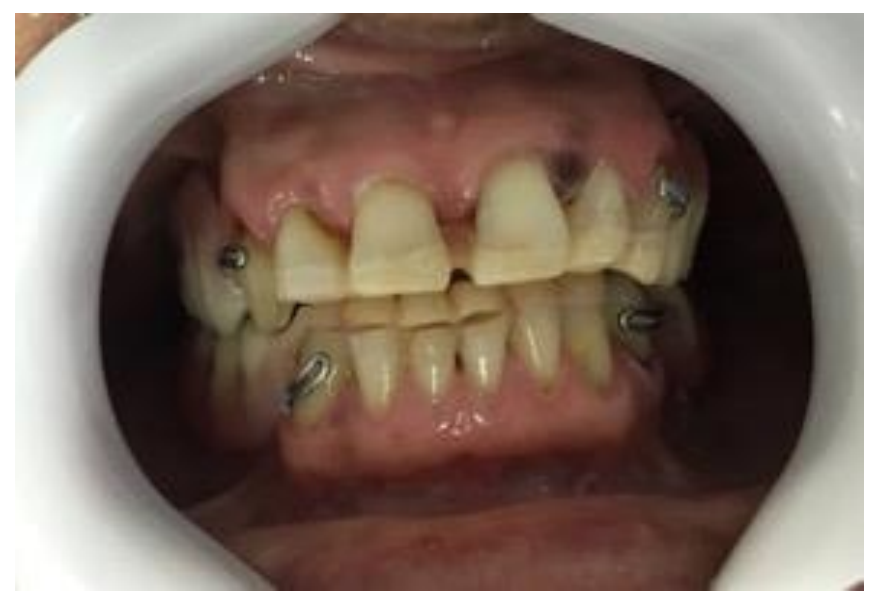

Figura 5 Foto intraoral mostrando vista frontal da prótese overlay antes do reembasamento final.

Os dentes que estavam desgastados devido a parafunção foram recobertos pela base da prótese com resina acrílica reconstruindo as incisais e inserindo dentes artificiais na região das ausências dentárias. A paciente foi orientada a fazer uso das próteses durante 30 dias e orientada quanto à correta higienização da nova prótese, esse tempo inicial de uso serviu para adaptação da nova DVO e; nas consultas de controle, foram realizados ajustes oclusais para equilíbrio neuromuscular. Passado os 30 dias a paciente já estava habituada com a nova DVO, e como consequência houve reestabelecimentos das relações intermaxilares e com isso proporcionando função mastigatória e conforto. A partir disso o modelo de estudo inicial foi entregue ao laboratório para enceramento com a finalidade de diminuir os diastemas com resina composta de forma direta. Ao receber os dentes Rev. Psicol Saúde e Debate. Jul., 2017:3(1):12-21. 
encerados foi feito moldagem com silicone de condensação que é indicada nesses casos em que há necessidade de reprodução de detalhes; a adaptação do molde aos dentes foi realizada com lâmina de bisturi para remover a porção vestibular. Foi feito isolamento relativo com rolos de algodão, condicionamento ácido do esmalte por 30 segundos no esmalte e 15 segundos em dentina com ácido fosfórico a 37\%, lavagem por 30 segundos e secagem completa com jato de ar, aplicação de sistema adesivo em duas camadas, e posteriormente a fotopolimerização. O molde de silicona foi preenchida com a resina, levada ao dente e fotopolimerizada, reproduzindo primeiramente as faces palatinas, após isso as faces incisais, mesiais e distais foram preeenchidas. Após isso foi feito ajuste das restaurações e acabamento e polimento após 7 dias. (Figuras 6 e 7)

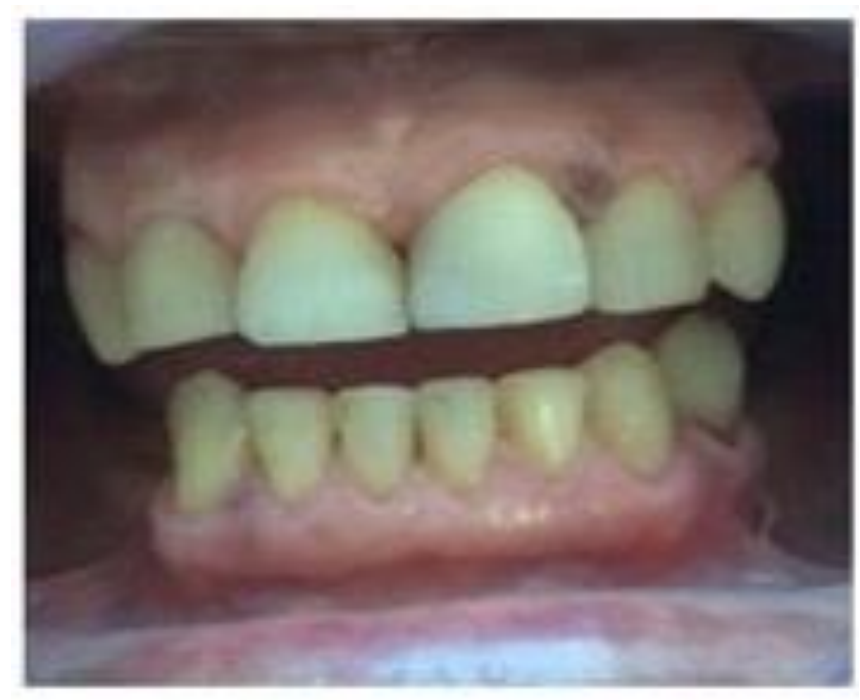

Figura 6 Foto intraoral mostrando as restaurações diretas em resinas compostas.

Após a acomodação da paciente à nova DVO e todos os dentes restaurados foi confeccionado uma prótese parcial removível "roach" para preencher as ausências dentárias, e posteriormente placa miorelaxante superior para controlar os sintomas e consequências do bruxismo. (Figura 8)

Rev. Psicol Saúde e 


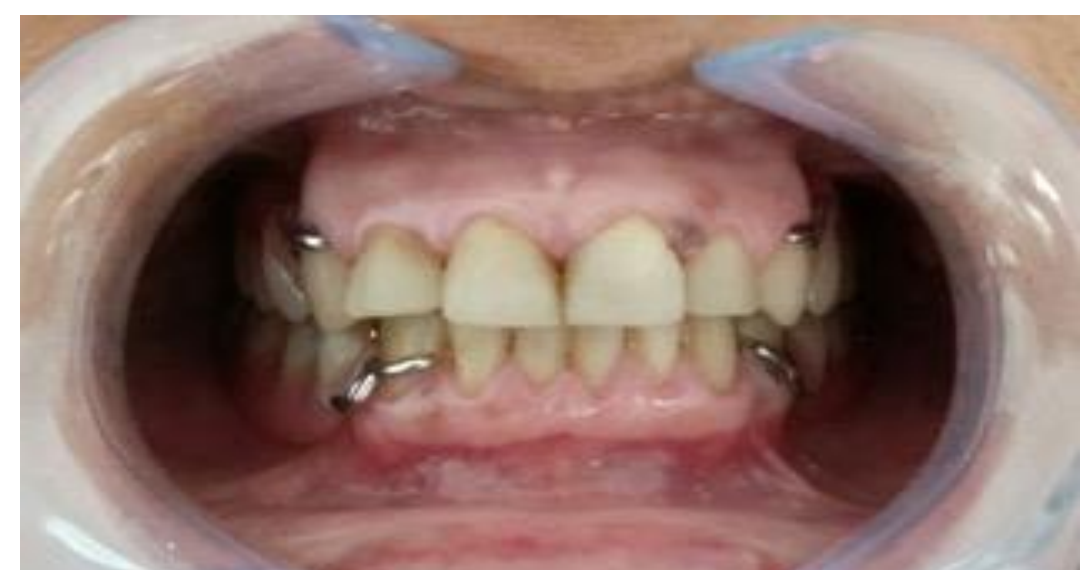

Figura 7 Foto intraoral mostrando as restaurações diretas em resinas compostas.

Figura 8 Foto mostrando as próteses acrilizadas instaladas e o tratamento finalizado.

\section{DISCUSSÃO}

Pacientes com DVO alterada são os que mais procuram atendimento protético, e na maioria dos casos o profissional não prioriza a reabilitação do sistema estomatognático comprometido, realizando somente a reposição dos dentes ausentes, podendo gerar ao longo do tempo iatrogenias irreversíveis ou de difícil resolução. A negligência em reabilitações realizadas sem critérios, conhecimento técnico prévio do cirurgião dentista e de um bom nível técnico-laboratorial, e planejamento prévio irá condenar todo o trabalho executado. Para que seja executada uma adequada reabilitação oral é necessário o restabelecimento da relação maxilomandibular, reparando, assim, a estética e função perdida. ${ }^{(1,4,7,9,10)}$

É necessário que seja restabelecido tanto a guia anterior como a guia canina em todo o tratamento reabilitador. O elemento de equilíbrio para todo complexo oclusal é a proteção mútua entre os segmentos posterior e anterior da cavidade oral, seja qual for a alteração nesse mecanismo de proteção mútua resultará a sérios danos, como o colapso oclusal, causando uma sobrecarga das forças mastigatórias nos dentes anteriores gerando reações como reabsorções, fraturas coronárias e/ou radiculares, desgaste da estrutura dentária o que promove necessidade restauradora. É mais complexo o restabelecimento da oclusão de pacientes parcialmente desdentados conforme o espaço protético aumenta, visto que a ausência dos dentes posteriores pode ocasionar alterações consideráveis na DVO. (4)

Rev. Psicol Saúde e Debate. Jul., 2017:3(1):12-21. 
As próteses parciais removíveis provisórias do tipo "overlay" devem ser utilizadas no início do tratamento, obtendo assim o restabelecimento de forma gradual da DVO, sendo de suma importância o uso dessas PPRs, podendo no final do tratamento o paciente não adaptar-se à nova DVO, acarretando insucesso do tratamento reabilitador. A utilização de próteses de recobrimento oclusal permitirão a adaptação progressiva á uma nova DVO, solucionando assim possíveis problemas funcionais. ${ }^{(4)}$

\section{CONCLUSÃO}

Foi possível deduzir que o bruxismo pode ter relação com a diminuição da DVO. O tratamento de escolha para o caso promoveu o restabelecimento de estética, conforto e função ao paciente. O restabelecimento da DVO foi necessário para o êxito do tratamento. O uso da prótese tipo overlay auxilia no restabelecimento inicial da DVO, possibilitando a reabilitação do paciente.

\section{REFERÊNCIAS}

1 Freitas R, Kaizer OB, Pigozo MN, Cavallari P, Resende DRB. Diagnóstico e tratamento da dimensão vertical de oclusão diminuída: utilização de prótese parcial removível atípica (PPR tipo “overlay"). RGO. 2005; 54(2): 161-164.

2 Silva MCVS, Carreiro AFP, Bonan RF, Carlo HL, Batista AUD. Reabilitação oclusal com prótese parcial removível provisória tipo "overlay": relato de caso. Rev. Bras. Ciênc. Saúde. 2011; 15(4): 455-460.

3 Rodrigues CK, Ditterich RG, Shintcovsk RE, Tanaka O. Bruxismo: Uma revisão de literatura. Publ. UEPG Ci. Biol. Saude. 2006; 12(3): 13-21.

4 Silva MPC, Girundi FMS. Restabelecimento da dimensão vertical de oclusão: relato de caso. [TCC] Belo Horizonte: Instituto de Estudos da Saúde; 2010.

5 Mukai MK, Gil C, Costa B, Stegun RC, Galhardo APM, Chaccur DC, Fukuda ACCS, Kammerer BA. Restabelecimento da dimensão vertical de oclusão por meio de prótese parcial removível. RPG Rev Pós Grad. 2010; 17(3): 1-6. 
6 Lara RA, Goiato NC, Fajardo RS, Santos DM, Moreno A, Tamae AC, Saavedra G, Rode SM. Parafunção Severa: reabilitação oral com prótese total mandibular x overlay Maxilar: relato de caso. Rev. Odontol. Araçatuba. 2012; 23(1): 37-40.

7 Amoroso AP, Gennari Filho H, Zuim PRJ, Mazaro JVQ, Zavanelli AC. Recuperação da dimensão vertical em pacientes com parafunção severa. Rev. Odontol. Araçatuba. 2013; 34(2): 9-13.

8 Souza JEA, Silva ET, Leles CR. Prótese parcial removível overlay: fundamentos clínicos e relatos de casos. Robrac.2009;18(47):41-48.

9 Pacheco AFR, Cardoso PC, Santos BMM, Ferreira MG, Monteiro LJA, Decurcio RA. Estratégia para restabelecimento de dimensão vertical de oclusão com mini-jig estético: relato de caso clinico. Rev Odontol Bras Central. 2012; 21 (56): 340-350

10 Waldolato A, Anéas GCG, Fonseca DR, Rocha RL. Má oclusão e hábitos bucais delet[erios: uma revisão crítica. Psicologia e Saúde em Debate. 2015; 1 (2): 35-45 